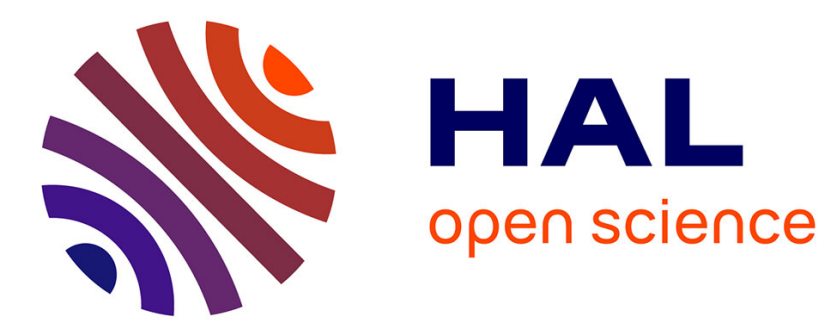

\title{
MEMS Four-Terminal Variable Capacitor for low power Capacitive Adiabatic Logic with High Logic State Differentiation
}

Hatem Samaali, Y. Perrin, A. Galisultanov, H. Fanet, Gaël Pillonnet, Philippe Basset

\section{To cite this version:}

Hatem Samaali, Y. Perrin, A. Galisultanov, H. Fanet, Gaël Pillonnet, et al.. MEMS Four-Terminal Variable Capacitor for low power Capacitive Adiabatic Logic with High Logic State Differentiation. Nano Energy, 2018, 10.1016/j.nanoen.2018.10.059 . hal-01910144

\author{
HAL Id: hal-01910144 \\ https://hal.science/hal-01910144
}

Submitted on 31 Oct 2018

HAL is a multi-disciplinary open access archive for the deposit and dissemination of scientific research documents, whether they are published or not. The documents may come from teaching and research institutions in France or abroad, or from public or private research centers.
L'archive ouverte pluridisciplinaire HAL, est destinée au dépôt et à la diffusion de documents scientifiques de niveau recherche, publiés ou non, émanant des établissements d'enseignement et de recherche français ou étrangers, des laboratoires publics ou privés. 


\title{
MEMS Four-Terminal Variable Capacitor for low power Capacitive Adiabatic Logic with High Logic State Differentiation
}

\author{
H. Samaali ${ }^{1}$, Y. Perrin ${ }^{2}$, A. Galisultanov ${ }^{2}$, H. Fanet ${ }^{2}$, G. Pillonnet ${ }^{2 *}$, P. Basset $^{1 *}$ \\ ${ }^{1}$ Université Paris-Est, ESYCOM, ESIEE Paris, Noisy-le-Grand 93160, France \\ ${ }^{2}$ Université Grenoble Alpes, CEA, LETI, F-38000 Grenoble, France \\ *Contact authors: philippe.basset@esiee.fr, gael.pillonnet@cea.fr
}

\begin{abstract}
This paper presents a novel four-terminal variable capacitor (FTVC) dedicated to the recent concept of low power capacitive adiabatic logic (CAL). This FTVC is based on silicon nano/micro technologies and is intended to achieve adiabatic logic functions with a better efficiency that by using field effect transistor (FET). The proposed FTVC consists of two capacitors mechanically coupled and electrically isolated, where a comb-drive input capacitor controls a gap-closing capacitor at the output. To fully implement the adiabatic combinational logic, we propose two types of variable capacitors: a positive variable capacitor (PVC) where the output capacitance value increases with the input voltage, and a negative variable capacitance (NVC) where the output capacitance value decreases when the input voltage increases. A compact and accurate electromechanical model has been developed. The electromechanical simulations demonstrate the ability of the proposed FTVC devices for CAL, with improved features such as high logic states differentiation larger than $50 \%$ of the full-scale input signal and cascability of both buffers and inverters. Based on the presented analysis, $89 \%$ of the total injected energy in the device can be recovered, the remaining energy being dissipated through mechanical damping. During one cycle of operation, a buffer gate of $10 \times 2.5 \mu \mathrm{m}^{2}$ dissipates only $0.9 \mathrm{fJ}$.
\end{abstract}

Keywords: Capacitive Adiabatic Logic, Ultra low power circuits, M/NEMS, Capacitive logic gates, Four Terminal Variable Capacitor (FTCV)

\section{Introduction}

A digital electronic circuit can be considered as a chain of interconnected elementary blocks [1], each of them having an input capacitance $C_{o}$ corresponding to the gate capacitor of a field effect transistor (FET) and some parasitic interconnections. In current CMOS technology, the classical combinational logic is based on the abrupt charge and discharge of $C_{o}$ through PMOS and NMOS transistors for coding a logic state, as in the CMOS inverter (Figure 1a). When changing from " 0 " to " 1 " logic state $\left(V_{\text {in }} \rightarrow 0\right), C_{o}$ is charged from the power supply $V_{C C}$ through the PMOS resistance in on-state $R_{o n}$, while the NMOS is in its cutoff region (Figure 1b). During this operation, the energy provided by the source is given by $Q V_{C C}=C_{o} V_{C C}{ }^{2}$ : half of it is dissipated through $R_{o n}$ and the other half is stored in $C_{o}$. When 
changing from " 1 " to " 0 " ( $\left.V_{\text {in }} \rightarrow 1\right), C_{o}$ is now discharged to the ground through the NMOS transistor in $\mathrm{ON}$ state (Figure 1c). The energy previously stored in $C_{o}$ is now dissipated into the ground and the total energy provided by the source is eventually fully dissipated.

In addition to the active power dissipation $C_{o} V_{C} C^{2} f$, where $f$ is the operating frequency, there are dissipation due to the short-circuit transition and the sub-threshold leakage $I_{\text {leak }} V_{C C}$, where $I_{\text {leak }}$ is the sub-threshold leakage current. The short-circuit transition power corresponds to about $10 \%$ of the average power and is typically the smallest contribution to the losses [3] [4][5]. Though among all VLSI logic families, CMOS circuits have the lowest power dissipation [2][3], reducing the energy consumption is one of the main concerns in modern integrated circuits (IC). It can be reduced by (i) developing devices with higher sub-threshold slope, (ii) reducing $C_{o}$ and (iii) working at low frequency. Reducing $C_{o}$ is limited by the lithography. Reducing the supply voltage $V_{C C}$ is a powerful method but this solution has limits related to the transistor size reduction [6]. Consequently, the dissipation cannot be reduced significantly.

In the early sixties, Landauer stated that to erase one bit of known information, which is consider as the most basic computing event, it requires the dissipation of at least $k_{B} \cdot T \cdot \ln (2)$, where $k_{B}$ is the Boltzman constant and $T$ is the temperature in Kelvin [7]. At room temperature, it corresponds to $18 \mathrm{meV}$. However, to overcome the energy barrier due to the thermal noise, a minimum of $100 . k_{B} . T\left(\sim 2.6 \mathrm{eV}\right.$ at $\left.300^{\circ} \mathrm{K}\right)$ is required [8]. As a comparison, the current dissipated energy at each switching event, with the $10 \mathrm{~nm}$ CMOS technology, is given by $0.5 C_{0} V_{c c}{ }^{2} \sim 40 \mathrm{eV}$, which is about 3 orders of magnitude above the Landauer limit and one order of magnitude above the thermal noise limit.

A good way to even further reduce the power consumption is the adiabatic logic approach [6][9], which can be combined with the concept of "energy recovery" [10]. It consists in charging the gate capacitor with a constant current, instead of a constant voltage as in classical digital circuits, then to recover the injected charge when going back to the initial state. It results in an optimized power dissipation [11] but requires a dynamic logic: the charge and discharge of $C_{o}$ have to be performed by a ramped supply voltage signal $V_{P C}$ called the Power Clock (PC).

\section{a) Dissipated energy in CMOS adiabatic logic}

The principle of the adiabatic logic is detailed in [6][9]. To explain the principle of operation of an adiabatic logic gate, let us consider the schematic of the inverter gate in Figure 1a. The PC signal is typically divided into 4 equal steps of equal duration $T$, that we named the Evaluate $(E)$, Hold $(H)$, Recovery $(R)$ and Wait $(W)$ intervals. Consequently, a logic "1" state at the input voltage $V_{\text {in }}$ is not a DC voltage value anymore as well. It corresponds to the power clock signal but shifted by a quarter of period $T$, as shown in Figure 1d.

During the charging phase (Evaluate stage), the supply voltage is ramped from 0 to $V_{C C}$ within the charging time $T$, and the current $i$ is determined by:

$$
0 \quad i=C_{o} \frac{d V_{P C}}{d t}=C_{o} \frac{V_{C C}}{T}
$$


The energy dissipated through $R_{o n}$ during the charging and discharging phases is then given by:

$$
0 \quad E_{\text {dynamic }}=2 i^{2} R_{\text {on }} T=2\left(\frac{C_{o} V_{C C}}{T}\right)^{2} R_{\text {on }} T=2 \frac{R_{o n} C_{o}}{T} C_{o} V_{C C}{ }^{2}
$$

If the time constant $2 R_{o n} C_{o}$ is lower than $T$, the energy dissipation is reduced compared to the energy dissipation $C_{o} V_{C C}{ }^{2}$ in classical CMOS circuits. The ramp duration of the PC (also equal to $T$ ) strongly affects the Joule dissipation: $E_{\text {dynamic }}$ vanishes for long charging times. Similarly, scaling down the supply voltage and reducing the capacitance load also reduce the dissipated energy in adiabatic logic, as we mentioned earlier.

In addition, it exits a non-adiabatic energy associated to the incompressible threshold voltage $V_{t h}$ of the MOS transistor equal to:

$$
0 \quad E_{\text {non-adiabatic }}=\frac{1}{2} C_{o} V_{t h}^{2},
$$

And a leakage current can flow from the supply voltage to the ground through the disable transistors due to the semiconductor technology, leading to a static energy dissipation given by:

$$
0 \quad E_{\text {static }}=\frac{\overline{\left(\left(I_{\text {leak }}\right)\right)} V_{C C}}{f}
$$

where $\overline{I_{\text {leak }}}$ is the mean leakage current.

By taking into account the (adiabatic) dynamic losses, the (static) leakage and the (uncompressible) non-adiabatic losses, we can express the total energy dissipation of an adiabatic inverter gate based on CMOS transistors as:

$$
0 \quad E_{\text {Total }}=2 \frac{R_{o n} C_{o}}{T} C_{o} V_{C C}{ }^{2}+\frac{1}{2} C_{o} V_{t h}^{2}+\overline{I_{\text {leak }}} V_{C C} \frac{1}{f}
$$

\section{b) Electromechanical devices to reduce the non-adiabatic losses}

Nano-Electromechanical relays (NEMS relays) have been proposed to replace CMOS transistors in order to reduce the static and non-adiabatic losses. Most of them are electrostatically actuated [12][13] but a nanomagnetic switch could also be used [8]. These nanorelays need low switching energy and promise perfect isolation with zero leakage current in the OFF state. They have already been evaluated for classical logic [14]-[15] and even tested with an integrated circuit [16]. In addition, they can be used efficiently for the adiabatic logic [17].

A classical NEMS relay has typically three terminals, the gate $(G)$, the source $(S)$ and the drain $(D)$ (Figure 1e). It consists in an electrostatic actuator made of a flexible beam $S$ and a fixed electrode $G$, forming a variable capacitance $C_{g}$ (Figure 1e). The voltage across $C_{g}$ determines the state of the relay. In the OFF state, $C_{g}$ is low and the resistance $R$ between the drain and the source is infinite (open circuit). When an input voltage is applied between $S$ and $G$, the beam deflects and creates a resistive contact between the drain and the source (ON 
states). Consequently, the relay can be seen as a capacitor controlling a variable resistor, represented with the symbol in Figure 1f. To implement all combinational logic, we need two types of relay: normally-ON and normally-OFF relays to replace the NMOS and PMOS transistors in integrated circuits respectively. Figure $1 \mathrm{~g}$ illustrates how the relays (or the MOS transistors) can be implemented in an adiabatic inverter.

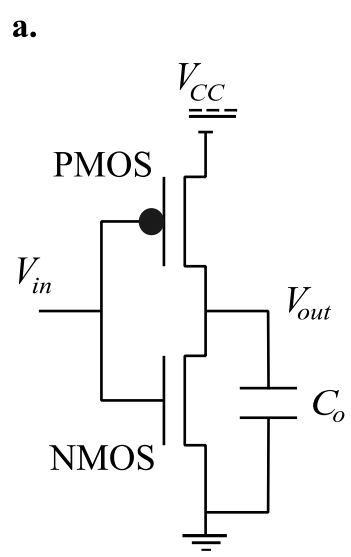

e.

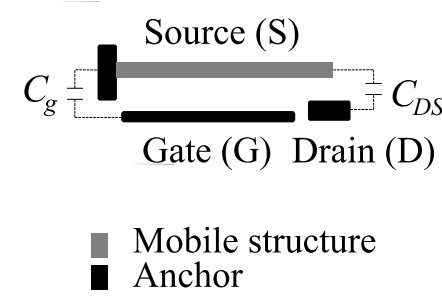

b.

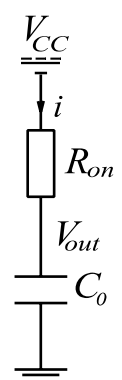

f.

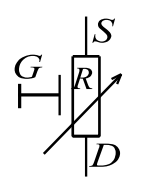

d.

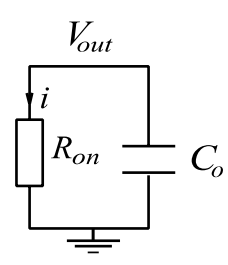

g.

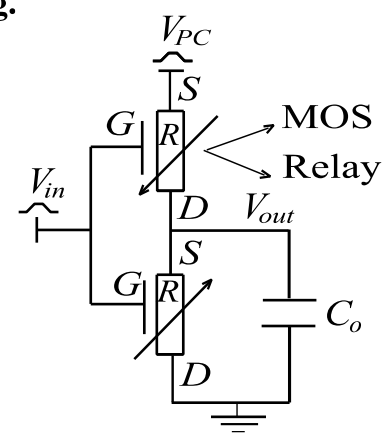

Figure 1. From classical CMOS inverter to inverter based on adiabatic logic. a) Schematic of a conventional CMOS inverter. b) Equivalent circuit in charging phase. c) Equivalent circuit in discharging phase. d) PC circuit signals. e) Implementation of a resistive electromechanical relay and f) its schematic representation. g) Schematics of an adiabatic resistive inverter.

However, the unavoidable contact resistance of resistive micro/nano-electromechanical relays, their poor mechanical reliability and their low switching frequencies have been highlighted in literature [12]-[17]. In order to avoid the resistive contact in the on-state and to reduce the dynamic dissipation, a purely capacitive electromechanical switch could be a better solution [18]. Based on this new paradigm, the so-called Capacitive Adiabatic Logic (CAL), Galisultanov et al have detailed in [19] the concept of a fully contact-less electromechanical device for the CAL. In this paper we described new four-terminal variable capacitors (FTVC) for the CAL that still have a contact zone but present several major advantages, like a better differentiation between a " 0 " and a " 1 " logic state, a higher frequency of operation or a smaller area. In addition, based on our simulations, we demonstrated for the first time the ability to cascade several devices. 
The organization of the paper is as follows. In the next section, we detail these new FTVC and their mathematical models that can be used to implement the CAL approach. In section 3, we analyze the different energy losses of the proposed configurations. We conclude with the demonstration of the operation of cascaded elementary combinational gates.

a.
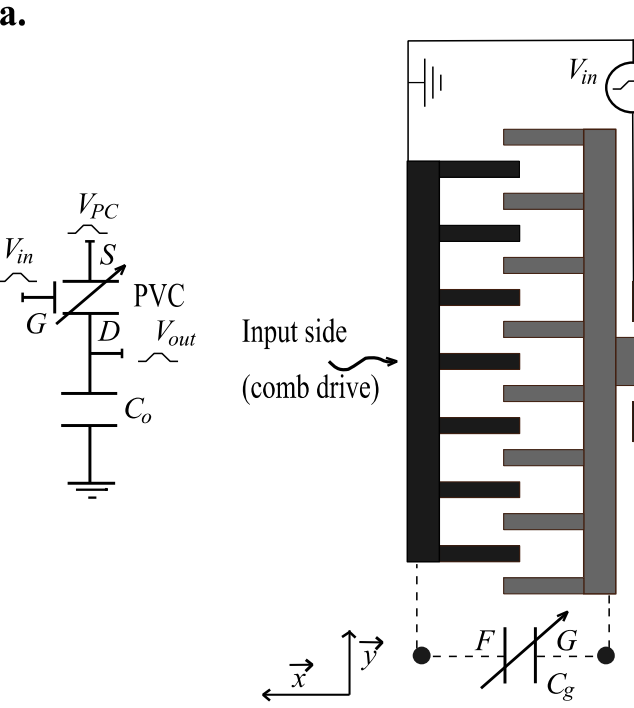
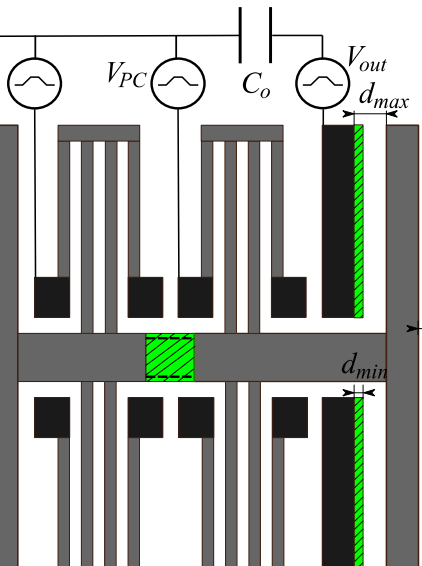

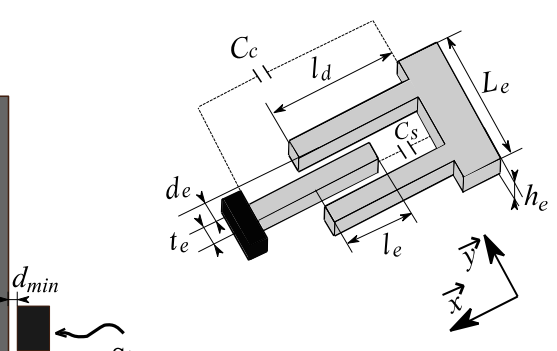

d.

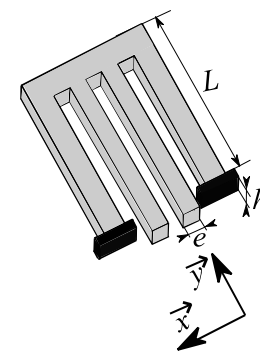

e.

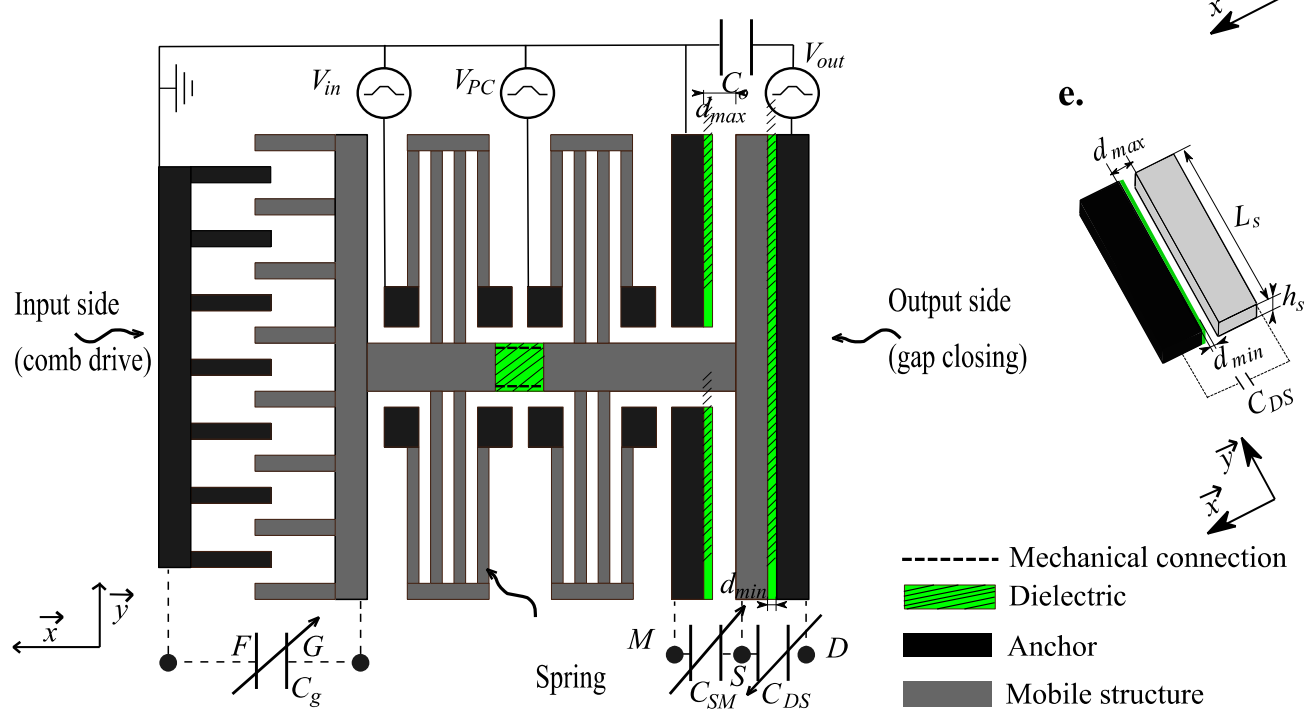

b.

Figure 2. Electromechanical implementations of FTVC in the OFF state. a) Electromechanical implementation of a buffer with a PVC. b) Implementation of an inverter with a NVC. c) Geometric details for the comb-drive capacitance $C_{g}$ for $n_{e}=1$. d) Geometric details for a spring. e) Geometric details for the gap-closing capacitance $C_{D S}$.

\section{Proposed architectures for the CAL devices}

\section{a) General description}

In the Capacitive Adiabatic Logic approach, the resistive elements (transistors or relays) are replaced by purely capacitive elements. Similarly to the resistive-based adiabatic logic, the 
logic function required an input capacitance $C_{g}$ between the gate $G$ and the ground, and an output capacitance $C_{D S}$ between the terminals $D$ and $S$ (we use the FET transistor notation, i.e. the input voltage $V_{\text {in }}$ is applied between the gate and the ground). The only difference is that the electrodes of $C_{D S}$ are never in ohmic contact. All the terminals are electrically isolated, but two of them $(G$ and $S$ ) are mechanically connected. Two types of output variable capacitances are needed to fully implement the adiabatic combinational logic: Positive Variable Capacitances (PVC) where $C_{D S}$ increases with the control voltage $V_{\text {in }}$ and Negative Variable Capacitances (NVC) where $C_{D S}$ decreases when $V_{\text {in }}$ increases.

In the following, we propose implementations for a PVC and a NVC that can be fabricated in silicon micro/nanotechnologies. Each device consists of an electrostatic comb-drive actuator for the input capacitance $C_{g}$ and a gap-closing (possibly with interdigited-combs) for the output capacitor $C_{D S}$. The capacitance $C_{g}$ and $C_{D S}$ are mechanically connected but electrically separated by a dielectric. Figure 2 a shows a schematic top-view of a practical buffer using a PVC and Figure $2 \mathrm{~b}$ depicts an inverter with a NVC, both in the OFF state. Figure 2c,d,e give more geometric details of $C_{g}$, the spring mechanical suspensions and $C_{D S}$ respectively.

\section{b) Principle of the PVC configuration}

Let's assume that the buffer in Figure 2a is loaded with the signals presented in Figure 1d. In the OFF state $\left(V_{i n}=0\right)$, the electrodes $S$ and $D$ are separated by $d_{\max }$, and the capacitance $C_{D S}$ is minimum (Figure 3a). When $V_{\text {in }}$ is applied (ON state), the comb-drive moves the rotor in the $\vec{x}$ direction, resulting in an increase of $C_{g}$ and $C_{D S}$. According to the capacitor divider $\left\{C_{o^{-}}\right.$ $\left.C_{D S}\right\}$, the output $V_{\text {out }}$ follows $V_{P C}$. The electrical force $F_{e}$, created by the voltage $V_{D S}=V_{P C}-V_{o u t}$, is opposed to the mechanical spring force $F_{m}$ (Figure 3b). Consequently, during the Hold interval when $V_{i n}$ decreases, $F_{e}$ balances $F_{m}$ and the equilibrium is maintained: $C_{g}$ and $C_{D S}$ remain constant, allowing $V_{\text {out }}$ to still follow $V_{P C}$.

\section{c) Principle of the NVC configuration}

Now we consider the inverter in Figure $2 b$ loaded with the same signals in Figure $1 \mathrm{~d}$. In the OFF state $\left(V_{i n}=0\right), C_{D S}$ is maximal because $S$ and $D$ are separated by $d_{\text {min }}$, the thickness of a dielectric layer covering the electrodes (Figure 3c). When $V_{\text {in }}$ is applied (ON state), the comb-drive moves the rotor in the $\vec{x}$ direction, resulting in an increase of $C_{g}$ and a decrease of $C_{D S}$. This time, the electrical force $F_{e}$ and spring force $F_{m}$ are in the same direction (Figure $3 \mathrm{~d})$. Consequenctly, during the Hold interval when $V_{\text {in }}$ decreases, the mobile electrode may return to its initial position causing an incorrect operation. To avoid this problem, we proposed to add an additional electrode $M$ connected to the ground and forming with $S$ a capacitance $C_{S M}$. The voltage across $C_{S M}$ creates an additional electrical force $F_{e}$ ' that balances the other forces and the equilibrium can be maintained (Figure 3e,f). 


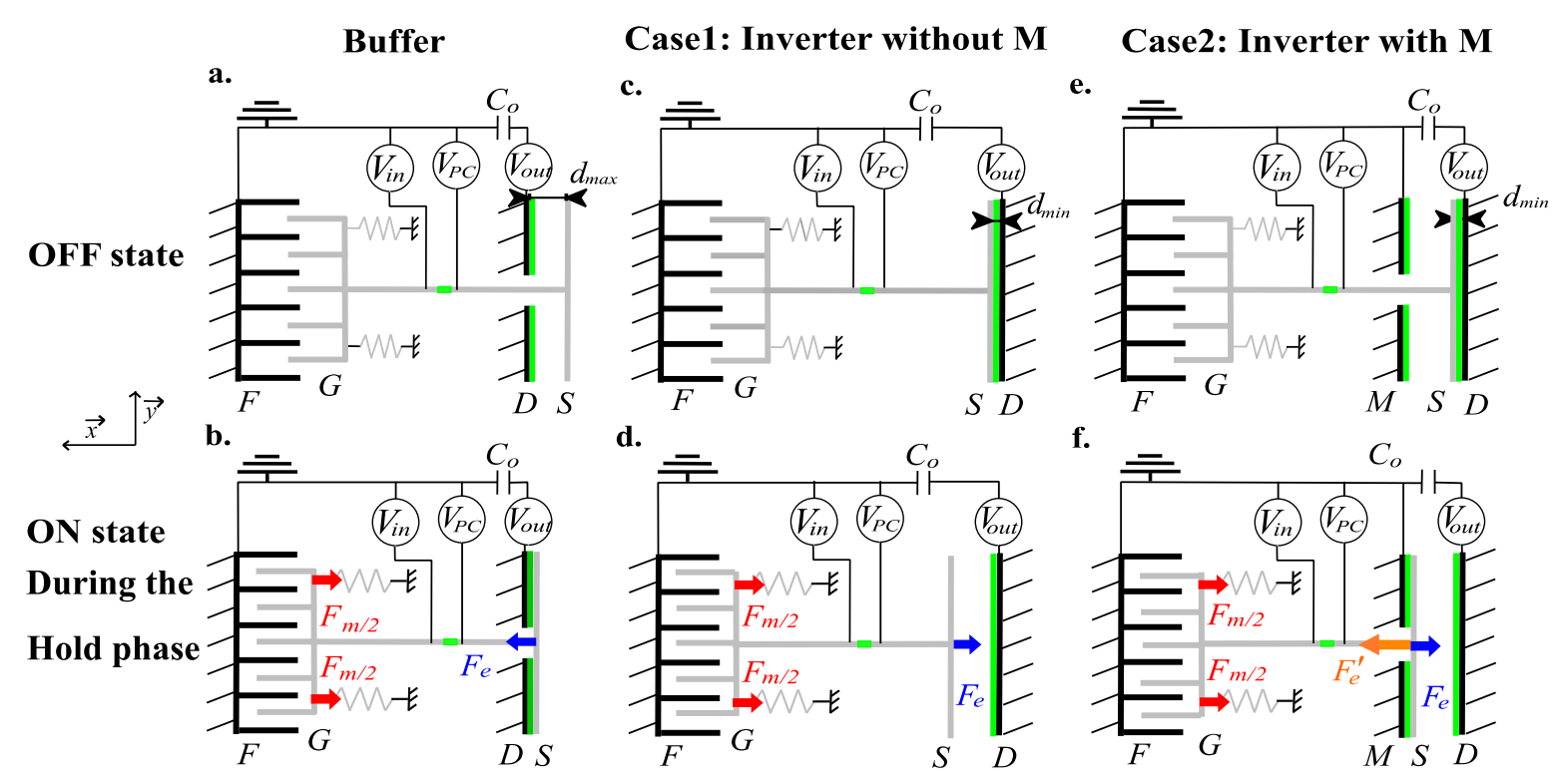

Figure 3. Different states of the buffer (PVC) and the inverter (NVC). a) Buffer in the OFF state. b). Buffer in the ON state during the Hold phase. c) Inverter without $M$ in OFF state. d) Inverter without $M$ in the ON state during the Hold phase. e) Inverter with $M$ in OFF state. f) Inverter with $M$ in the ON state during the Hold phase.

\section{d) The adiabatic conditions}

\section{Conditions related to input signals}

In order to satisfy the adiabatic conditions, the inputs have to be stable during ramp-up and ramp-down phases of the $\mathrm{PC}$, and the durations of the Evaluate, Hold, and Wait phases of both input and power-clock signals have to be equal [20][21]. Therefore, as already stated in the introduction, a four-phase power clock consisting in four equal intervals $T$ is required, and the input signal is shifted by a quarter of period with respect to the PC. In the ON state, the following rules have to be followed :

(i) During the Wait interval, $V_{\text {in }}$ ramps up, $C_{g}$ increases and reaches its maximum value at the end of the interval. As soon as $C_{g}$ increases, $C_{D S}$ evolves depending on the gate configuration: for a PVC, it increases until its maximum and for the NVC, it decreases to its lower value.

(ii) During the Evaluate interval, $V_{P C}$ increases and $V_{\text {in }}$ remains constant at its high value. In the PVC configuration, the output $V_{\text {out }}$ follows the PC and the capacitance node $C_{o}$ starts to charge. In the NVC, the output remains at a low level.

(iii) During the Hold interval, $V_{\text {in }}$ decreases but $V_{P C}$ remains high, and then the variables capacitances $C_{g}$ and $C_{D S}$ doesn't change (thanks to the help of electrode M for the NVC).

(iv) During the Recover interval, $V_{P C}$ decreases while $V_{\text {in }}$ remains grounded. The mechanical equilibrium is holded until low value of $V_{P C}$, thanks to the typical hysteresis in electrostatic transducers [23]. Both $C_{g}$ and $C_{D S}$ remain constant till then, and the charge stored in $C_{o}$ can be recovered by the source. 
To build a circuit, the CALs gates are cascaded and each gate is supplied by a PC, as shown in Figure S1. Since the output signal follows the PC, two subsequent PC should be delayed by a quarter of period to satisfy the adiabatic conditions. Therefore, a four-phase power-clock ( $V_{P C 1}$ to $\left.V_{P C 4}\right)$ is needed to cascade several gates.

\section{Conditions related to $C_{D S}$}

Figure 4 shows schematic representations of a buffer and an inverter. The PVC design has four terminals while NVC has five, since it includes the additional electrode $M$. The electrical equations are given by:

$$
\begin{gathered}
0 V_{P C}=V_{D S}+V_{\text {out }} \\
0 \quad i=C_{D S} \frac{d V_{D S}}{d t}+V_{D S} \frac{d C_{D S}}{d t}=C_{o} \frac{d V_{\text {out }}}{d t}
\end{gathered}
$$

where $V_{D S}, C_{o}$, and $i$ are the voltage across the drain and source of either PVC or NVC, the input capacitance of the following gate and the current provided by the power clock respectively. We can deduce the instantaneous logic output voltage as:

$$
0 \quad V_{\text {out }}=V_{P C} \frac{C_{D S}}{C_{D S}+C_{o}}
$$

Since the input voltage $V_{\text {in }}$ controls the value of $C_{D S}$, it also controls the voltage ratio between the power-clock signal and the output node through the capacitor bridge divider $\left\{C_{D S}-C_{o}\right\}$. The values of $C_{D S}$ have to be carefully chosen: the minimum value of $C_{D S}$ has to be small compared to $C_{o}$ in order to obtain the low logic state, and the maximum value of $C_{D S}$ has to be high compared to $C_{o}$ to achieve the high logic level.

a.

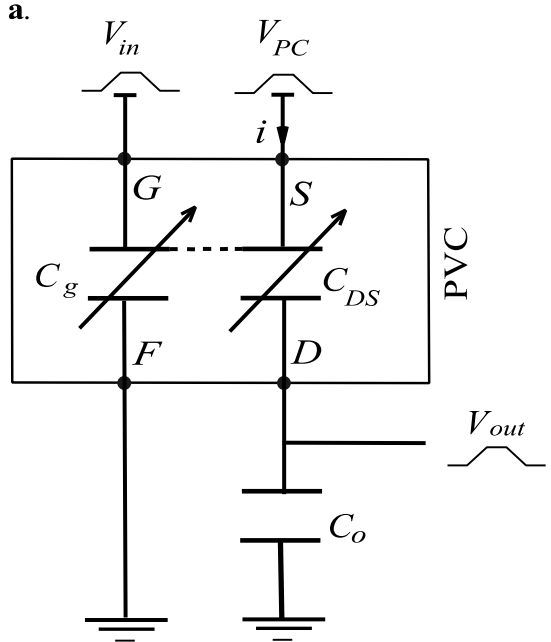

b.

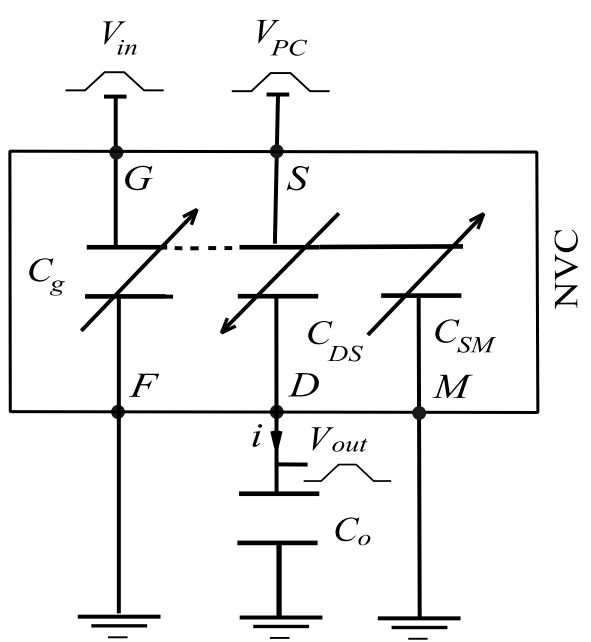

Figure 4. Equivalent circuit of a) a CAL buffer, and b) a CAL inverter. 


\section{e) Modelling the CAL devices}

\section{Modeling the input capacitance variation}

Various models can be found in the literature to model capacitances and their fringe fields. In this work, we use the Mejis-Fokkema formula [22] for the comb-drive $C_{g}$ and the Palmer formula [24] for the gap-closing capacitances $C_{D S}$ and $C_{S M}$.

For $C_{g} 0$, the first term $C_{c}$ represents the lateral capacitance without fringing effect, $C_{f}$ estimates the fringe capacitance and $C_{S}$ is the capacitance at the tip of fingers (c.f. Figure 2c):

$$
\begin{gathered}
0 C_{g}=2 C_{c}+C_{f}+C_{s} \\
0 \quad C_{g}=\frac{2 n_{e} \varepsilon h_{e}\left(l_{e}+x\right)}{d_{e}}+2 n_{e} \varepsilon h_{e}\left[0.77+1.06\left(\frac{l_{e}+x}{d_{e}}\right)^{1 / 4}+1.06\left(\frac{h_{e}}{d_{e}}\right)^{1 / 2}\right]+\frac{2 n_{e} \varepsilon h_{e} t_{e}}{l_{d}-l_{e}-x}
\end{gathered}
$$

where $n_{e}$ and $h_{e}$ represents the comb's finger numbers and their thickness respectively. $l_{e}, d_{e}$, $l_{d}$ and $t_{e}$ are the initial overlapping distance between the fingers, the initial gap distance between the fixed and movable fingers, the length and width of the finger respectively. $\varepsilon$ is the air permittivity and $x$ is the displacement in the $\vec{x}$ direction. This model is in good agreement with the commercial software MEMS+ from Coventorware (cf Figure S2)

\section{Modeling the ouput capacitance variation}

The gap-closing capacitance $C_{D S}$ is given by the Palmer formula [24], 0 and 0 for PVC and NVC respectively:

$$
\begin{aligned}
& 0 \quad C_{D S_{P V C}}=\frac{n_{s} h_{S} L_{s} \varepsilon}{d_{\max }-x}\left[1+2\left(\frac{d_{\max }-x}{h_{s} \pi}\right)\left(1+\log \left[\frac{h_{S} \pi}{d_{\max }-x}\right]\right)\right] \\
& 0 \quad C_{D S_{N V C}}=\frac{n_{S} h_{S} L_{S} \varepsilon}{d_{\min }+x}\left[1+2\left(\frac{d_{\min }+x}{h_{S} \pi}\right)\left(1+\log \left[\frac{h_{s} \pi}{d_{\min }+x}\right]\right)\right]
\end{aligned}
$$

The expression of $C_{S M}$ is similar to the expression of $C_{D S}$ in $\mathrm{PVC}$, and is expressed by:

$$
0 C_{S M}=C_{D S_{P V C}}=\frac{n_{S} h_{S} L_{S} \varepsilon}{d_{\max }-x}\left[1+2\left(\frac{d_{\max }-x}{h_{s} \pi}\right)\left(1+\log \left[\frac{h_{S} \pi}{d_{\max }-x}\right]\right)\right]
$$

where $n_{s}, h_{s}, L_{s}, d_{\max }$ or $d_{\min }$ are the number of $C_{D S}$ electrodes, their thickness, their length and their initial gap distance respectively.

Table 1. Non-dimensional variables

\begin{tabular}{|c|c|}
\hline Variable & Description \\
\hline$\hat{x}=x / d_{e}$ & Displacement in the direction $\vec{x}$ \\
\hline$\hat{t}=t \sqrt{k_{x} / m}$ & Time \\
\hline$\lambda=c / \sqrt{m \cdot k_{x}}$ & Damping \\
\hline$\beta_{1}=\left(l_{d}-l_{e}\right) / d_{e}$ & Gap of the side capacitance $C_{s}$ \\
\hline$\beta_{2}=l_{e} / d_{e}$ & Overlapping distance between fingers \\
\hline
\end{tabular}




\begin{tabular}{|c|c|}
\hline$\beta_{3}=d_{\max } / d_{e}$ & Initial gap of PVC $C_{D S}$ \\
\hline$\beta_{5}=d_{\min } / d_{e}$ & Initial gap of NVC $C_{D S}$ \\
\hline$\hat{V}_{i n}=\mu V_{i n}$ & Input signal, where $\mu=\sqrt{\frac{\varepsilon n_{e} h_{e}}{k_{x} d_{e}^{2}}}$ \\
\hline$\hat{V}_{P C}=\mu V_{P C}$ & Power Clock signal \\
\hline$\hat{V}_{D S}=\mu V_{D S}$ & Signal across the output capacitance $C_{D S}$ \\
\hline$\hat{V}_{C C}=\mu V_{C C}$ & Maximal supply voltage of different signal \\
\hline$\widehat{E}_{m=} E_{m} / \alpha_{1}$ & Mechanical energy where $\alpha_{1}=k_{x} d_{e}^{2}$ \\
\hline$\hat{E}_{i n}=E_{\text {in }} \mu^{2} / C_{o}$ & Input energy produced by the input source where \\
\hline$\hat{E}_{C g}=E_{C g} \mu^{2} / C_{o}$ & Electrical energy stored in the input capacitance $C_{g}$ \\
\hline$\widehat{E}_{P C}=E_{P C} \mu^{2} / C_{o}$ & Electrical input energy produced by the Power clock \\
\hline$\hat{E}_{C T}=E_{C T} \mu^{2} / C_{o}$ & Electrical energy stored in $C_{D S}$ and $C_{o}$ \\
\hline$\hat{C}_{g}=C_{g} / C_{o}$ & Input capacitance \\
\hline$\hat{C}_{D S}=C_{D S} / C_{o}$ & Output capacitance \\
\hline
\end{tabular}

Table 2. Physical and geometric parameters used in simulations.

\begin{tabular}{|c|c|c|}
\hline Variable & Description & Value \\
\hline$E$ & Yong's modulus of silicon $\left(\mathrm{N} / \mathrm{m}^{2}\right)$ & $16910^{9}$ \\
\hline$\varepsilon$ & Permittivity of air $\left(\mathrm{F} / \mathrm{m}^{3}\right)$ & $8.85410^{-12}$ \\
\hline$L_{s}$ & Length of the gap closing capacitance $C_{D S}(\mu \mathrm{m})$ & 8.72 \\
\hline$h_{s}$ & Thickness of the gap closing capacitance $C_{D S}(\mu \mathrm{m})$ & 0.4 \\
\hline$h_{e}$ & Thickness of the comb-drive capacitance $C_{g}(\mu \mathrm{m})$ & 0.4 \\
\hline$d_{\max }$ & Initial gap distance of $C_{D S}(\mu \mathrm{m})$ & 0.25 \\
\hline$d_{\min }$ & Thickness of the dielectric $(\mu \mathrm{m})$ & 0.01 \\
\hline$n_{s}$ & Number of the gap-closing capacitance $C_{D S}$ & 1 \\
\hline$n_{e}$ & Number of finger in $C_{g}$ & 55 \\
\hline$L$ & Length of the mechanical suspension $(\mu \mathrm{m})$ & 5 \\
\hline$e$ & Width of mechanical suspension $(\mu \mathrm{m})$ & 0.02 \\
\hline$h$ & Thickness of mechanical suspension $(\mu \mathrm{m})$ & 0.4 \\
\hline$L_{e}$ & Length of the comb-drive capacitance $C_{g}(\mu \mathrm{m})$ & 8.8 \\
\hline$d_{e}$ & Gap distance of the comb-drive capacitance $C_{g}(\mu \mathrm{m})$ & 0.04 \\
\hline$t_{e}$ & Width of the finger of the comb-drive capacitance $C_{g}(\mu \mathrm{m})$ & 0.04 \\
\hline$l_{e}$ & Overlapping distance between fingers of $C_{g}(\mu \mathrm{m})$ & 0.02 \\
\hline$l_{d}$ & Length of the finger of the comb-drive capacitance $C_{g}(\mu \mathrm{m})$ & 0.4 \\
\hline$m$ & Effective mass of the movable part $(\mathrm{Kg})$ & $1.9810^{-14}$ \\
\hline$K_{x}$ & Spring constant in the direction $\vec{x}(\mathrm{~N} / \mathrm{m})$ & 0.02 \\
\hline$c$ & Damping $(\mathrm{Kg} / \mathrm{s})$ & $1.9610^{-8}$ \\
\hline$C_{0}$ & Load capacitance $(\mathrm{fF})$ & 1 \\
\hline
\end{tabular}

\section{Modeling the device dynamics}

From the Newton's second law, the dynamic behavior of the PVC and NVC structures is represented by the non-dimensional equation 0 . The first term is associated to the kinetic force, the second term is associated to the mechanical damping force, the third one is related to the spring force, the fourth one represents the electrical force from $C_{g}$ and the next one is the electrical force from $C_{D S}$. The last term represents the electrical force associated to $C_{S M}$ for the NVC structure. The non-dimensional variables used in 0 are given in Table 1. 


$$
\begin{gathered}
0 \quad \ddot{\hat{x}}+\lambda \dot{\hat{x}}+\hat{x}-\left(\hat{V}_{\text {in }}\right)^{2}\left(1+\frac{1}{\left(\beta_{1}-\hat{x}\right)^{2}}+\frac{\gamma}{\left(\beta_{2}+\hat{x}\right)^{0.75}}\right) \\
+\alpha\left[\alpha_{1} \widehat{V}_{D S}^{2}\left(\frac{1+\beta_{4}\left(\beta_{5}+\hat{x}\right)}{\left(\beta_{5}+\hat{x}\right)^{2}}\right)-\alpha_{2}\left(\hat{V}_{P C}\right)^{2}\left(\frac{1+\beta_{4}\left(\beta_{3}-\hat{x}\right)}{\left(\beta_{3}-\hat{x}\right)^{2}}\right)\right]=0
\end{gathered}
$$

where $\gamma=0.265, \beta_{4}=\frac{2 d_{e}}{h_{s}}, \alpha=\frac{n_{s} h_{s} L_{s}}{2 n_{e} h_{e} d_{e}}, \alpha_{1}=-1$ and $\alpha_{2}=0$, for PVC configuration and $\alpha_{1}=\alpha_{2}=1$ for NVC.

\section{Modeling the impact}

At each impact between the drain and the source electrodes, the mobile electrode losses a small part of its kinetic energy during a bouncing phenomenon. During successive contacts, the velocity can be expressed as [25][26]:

$$
0 \dot{\hat{x}}_{n}=-K_{\text {loss }} \dot{\hat{x}}_{n-1}
$$

where $\dot{\hat{x}}_{n-1}$ and $\dot{\hat{x}}_{n}$ are the velocities before and after the $n^{\text {th }}$ impact, and $K_{\text {loss }}$ is the coefficient of restitution. When the mobile electrode reaches the contact, its velocity is set according to 0 . The characteristics of the bounces depend on the mass of the mobile part and its velocity, the elastic response of the material and the surface hardness [26].

\section{Simulations and analysis}

In this section, we present and analyze the simulations of Capacitive Adiabatic Logic circuits using the MEMS devices presented previously. The physical and geometrical features of the proposed implementations are given in Table 2. These dimensions are carefully chosen in order to match the FET transistor parameters. First, we present how a buffer or an inverter works. Then we study the energy transfer and losses in a buffer gate. Finally, we demonstrate the possibility to cascade in series several buffers or inverters.

In the following, a "1" adiabatic logic input (i.e. the "ON state") corresponds to a 4interval pulse similar to $V_{P C}$, but with a phase shift of a quarter of period in order to satisfy the adiabatic logic conditions. A " 0 " ("OFF state") means $V_{i n}=0$ during the entire sequence (Figure 1d). 


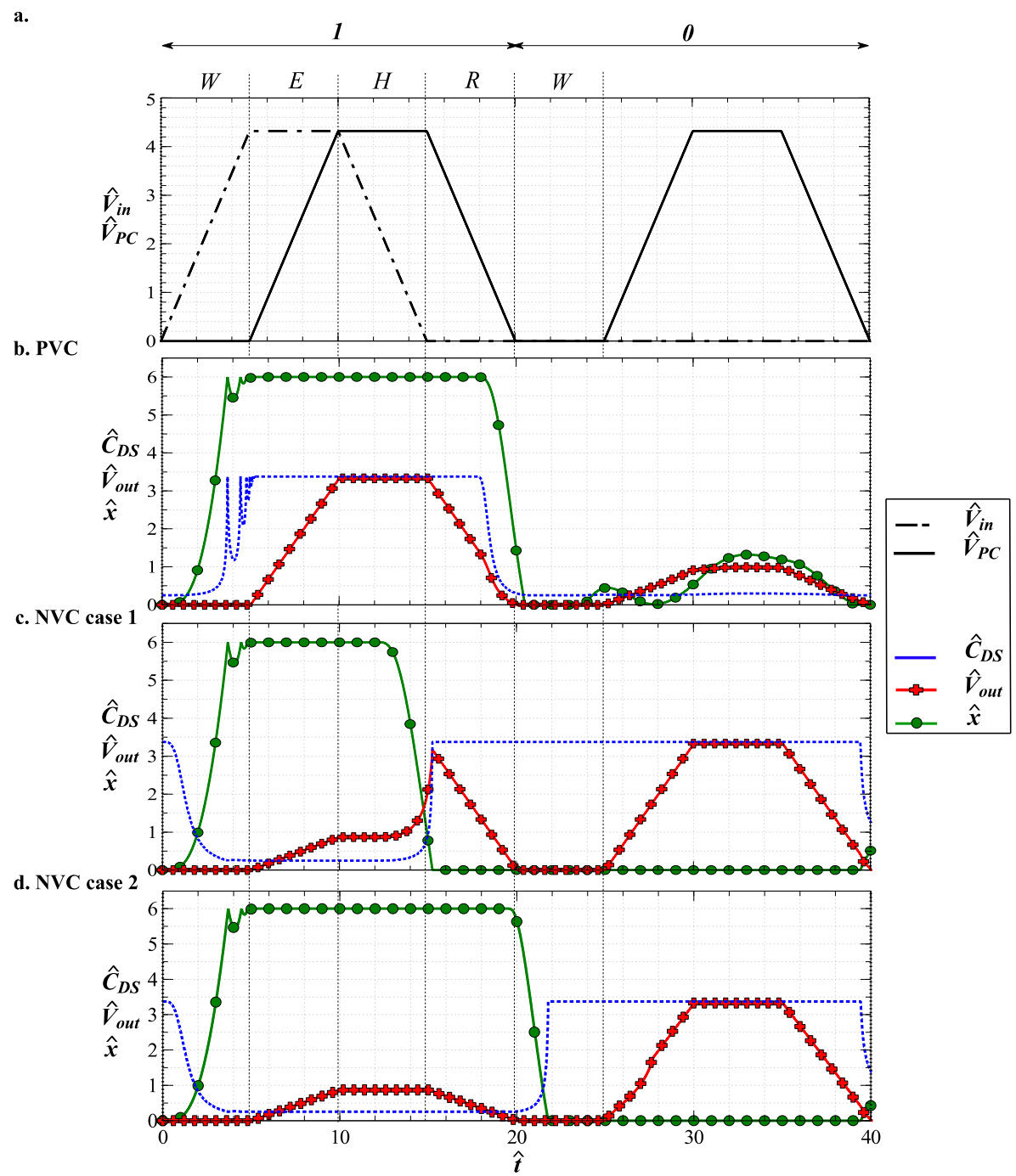

Figure 5. Operation of simple gates for a "1 0 " logic input. a) the input and PC signals. b) Simulation of a CAL buffer. c) Simulation of a CAL inverter without electrode $M$ (case 1) and d) with electrode $M$ (case 2). All dimensions are given in Table 1.

\section{a) Analysis of a single gate}

\section{Simulation of a buffer}

In order to understand how a CAL buffer works, we plotted in Figure $5 b$ the output voltage of a PVC and the displacement of its mobile part for the input logic sequence " 10 " shown in Figure 5a. $C_{o}$ is set to $1 \mathrm{fF}$ according to [1]. During the "1" state, as soon as $V_{\text {in }}$ increases (Wait interval), the comb-drive at the input moves the mobile part, $d_{s}=d_{\max }-x$ decreases and $C_{D S}$ increases from its minimum to its maximum value, with a short oscillation induced by the impact of the mobile mass onto the rigid frame. Then, during the Evaluate interval, $V_{i n}$, and so $C_{D S}$ are constant, while $V_{P C}$ increases. $C_{D S}$ is at its maximum value and $V_{\text {out }}$ follows $V_{P C}$ through the constant capacitive divider formed by the $\left\{C_{D S}-C_{o}\right\}$ couple. This lasts even beyond the Hold interval, while $V_{\text {in }}$ decreases till $0 \mathrm{~V}$, because of the electrostatic force $F_{e}$ across $C_{D S}$ introduced by $V_{P C}$ is still higher than the restoring force of the mechanical springs $F_{m}$. This memory effect corresponding to the hysteresis of electrostatic transducers [23] is 
important to keep $C_{D S}$ at its maximum value, allowing $V_{\text {out }}$ to follow the PC. During the Recovery interval, $V_{\text {out }}$ progressively decreases with $V_{P C}$, and at some point the electrostatic force associated to $C_{D S}$ is not sufficient to compensate the restoring spring force, so $C_{D S}$ rapidly decreases to its minimum value, bringing $V_{\text {out }}$ definitely to $0 \mathrm{~V}$.

Then the " 0 " state starts. $V_{\text {in }}$ remains equal to 0 during the 4 intervals of $V_{P C}$ and the electrostatic force across $C_{g}$ is null. However, when $V_{P C}$ increases during the Evaluate interval, its associated electrostatic force leads to a small variation of $C_{D S}$ that is not negligible compare to $C_{o}$, so we can observe a small variation of $V_{\text {out }}$ following the evolution of $V_{P C}$. In addition, we can also observe a tiny increase of $C_{D S}$ due to the electrostatic force across $C_{D S}$ introduced by $V_{P C}$. However $V_{\text {out }}$ has to remain below the threshold voltage corresponding to the " 0 " logic state.

\section{Simulation of an inverter}

Similarly, the time history of a NVC loaded with a capacitor $C_{o}$ of $1 \mathrm{fF}$ is given in Figure $5 \mathrm{c}$ and $\mathrm{d}$ for the same " 10 " logic input. At $t=0, C_{D S}$ is at its maximum and $V_{\text {in }}$ starts to increase from $0 \mathrm{~V}$ to $V_{C C}$ (Wait interval). $V_{\text {out }}$ is initially equal to $0 \mathrm{~V}$, so the mobile electrode easily moves with the increase of $V_{i n}$, and $C_{D S}$ quickly decreases to its minimum value. During the Evaluate interval, $V_{i n}$ and so $C_{D S}$ remain constant. Since $V_{P C}$ increases and $C_{D S}$ is not negligible, $V_{\text {out }}$ follows $V_{P C}$ but has to remain below the maximum voltage allowed for a "0" logic level. During the following Hold interval, $V_{\text {in }}$ decreases. First $C_{D S}$ remains at its minimum because the electrostatic force across $C_{g}$ is still higher that the spring restoring force. However, at some point, this is not the case anymore. Then $C_{D S}$ increases, while $V_{P C}$ is still high, which leads to an unexpected increase of $V_{\text {out }}$ before the next " 0 " logic at the input (case 1 Figure 5c). To avoid this, we added the $M$ electrode connected to the ground that creates an additional electrostatic force across $C_{S M}$, controlled by $V_{P C}$ and opposed to the spring force (Figure $3 \mathrm{e}, \mathrm{f}$ ). Hence, $C_{D S}$ can be maintained at its minimum value during the whole Hold interval (case 2 Figure $5 \mathrm{~d}$ ).

Close to the end of the Recover interval, while $V_{P C}$ is small enough, the mobile part progressively moves back to its initial position. Simultaneously $C_{D S}$ progressively gets back to its maximum value, which is reached at the beginning of the Wait interval. Hence $V_{\text {out }}$ is always bellow the " 1 " logic state before the end of the " 1 " sequence at the input. 
a.

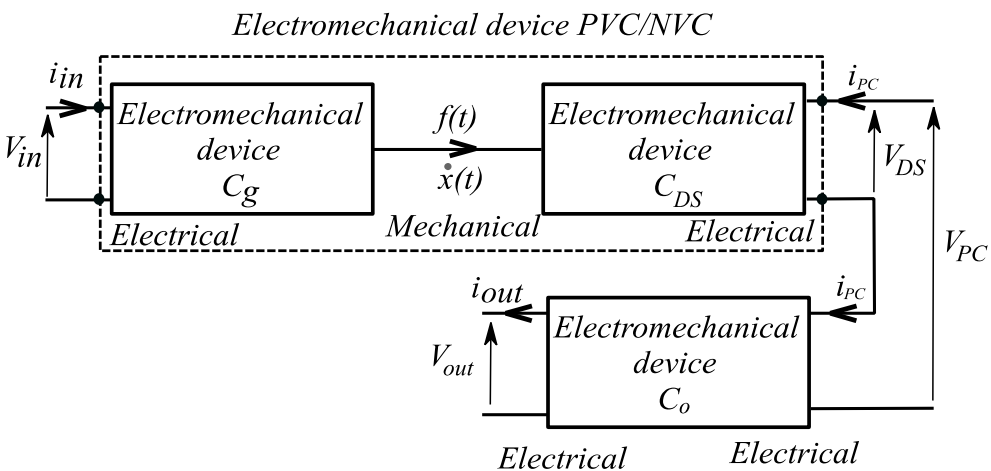

b.

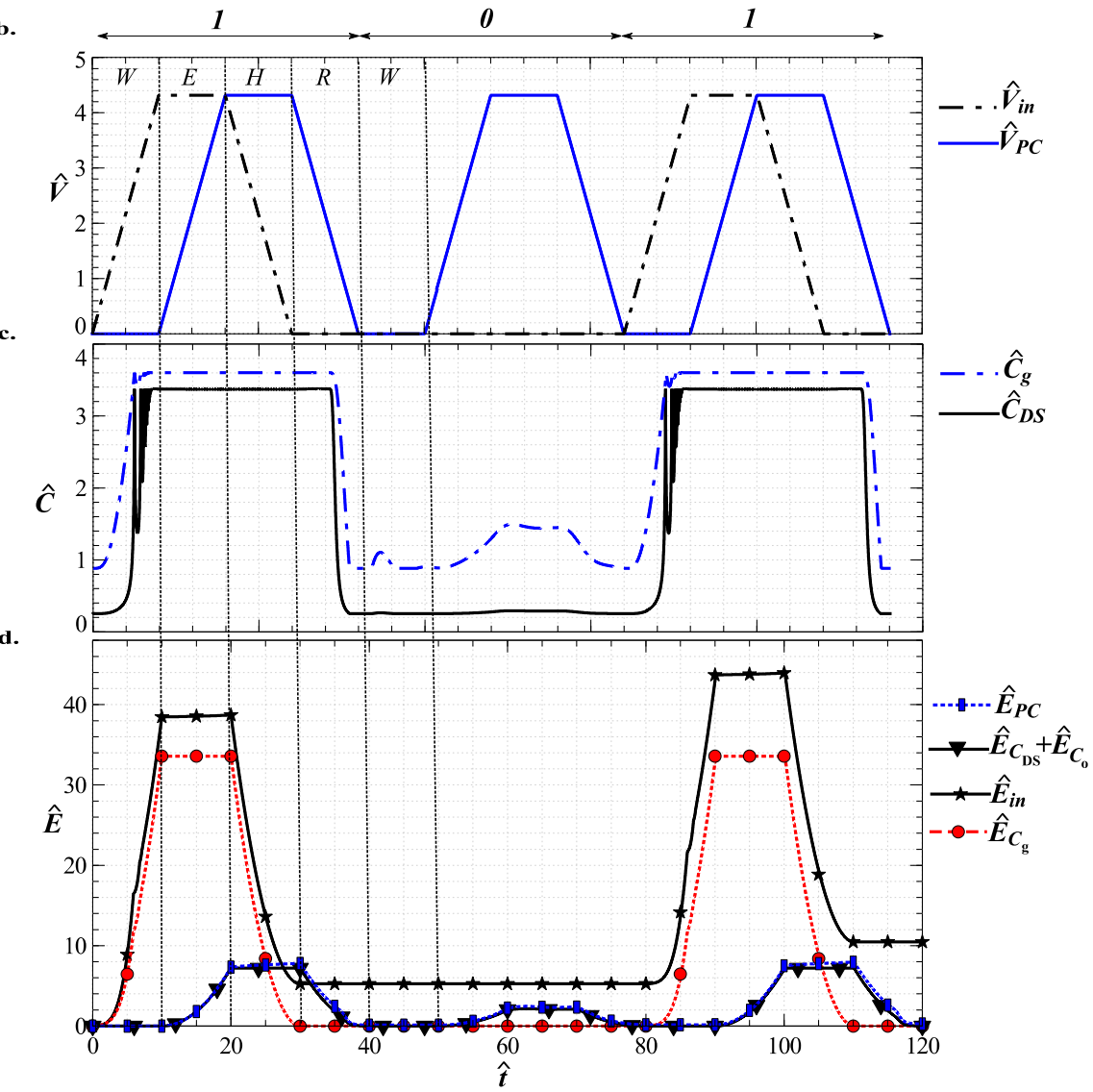

Figure 6. Energy Analysis of a buffer. a) Block-schematic representation of a gate (PVC or NVC). b) Evolution a the buffer's input voltages. c) Evolution of capacitances $\hat{C}_{g}$ and $\hat{C}_{D S}$. d) Energy contributions of electrical parts.

\section{b) Energy analysis}

In this section, we investigate the energy balance of the design and we show that only a small part of the energy provided by the electrical sources cannot be recovered.

$C_{g}$ and $C_{D S}$ are two electromechanically coupled transducers having two electrical inputs and one mechanical output (Figure 6a). The instantaneous electrical energy is given by:

$$
\text { (Eq 16) } E_{e}(t)=E_{\text {in }}+E_{P C}=\int\left(V_{\text {in }} i_{\text {in }}+V_{P C} i_{P C}\right) d t
$$




$$
=\int\left[\mathrm{V}_{\text {in }} i_{\text {in }}+\left(V_{D S}+V_{\text {out }}\right) i_{P C}\right] d t
$$

The instantaneous mechanical energy output of the system is given by:

$$
\text { (Eq 17) } E_{m}(t)=\int f(t) \dot{x}(t) d t
$$

where $f(t)$ is the mechanical force applied on the mobile part and $\dot{x}(t)$ is its velocity. The expression of $i_{\text {in }}$ and $i_{P C}$ are given by:

$$
\text { (Eq 18) } i_{\text {in }}=\frac{d\left(C_{g} V_{i n}\right)}{d t}=C_{g} \frac{d V_{i n}}{d t}+\frac{d C_{g}}{d x} V_{\text {in }} \frac{d x}{d t}=C_{g} \frac{d V_{i n}}{d t}+\frac{d C_{g}}{d x} V_{i n} \dot{x}(t),
$$

(Eq 19) $i_{P C}=\frac{d\left(C_{D S} V_{D S}\right)}{d t}=C_{D S} \frac{d V_{D S}}{d t}+\frac{d C_{D S}}{d x} V_{D S} \frac{d x}{d t}=C_{D S} \frac{d V_{D S}}{d t}+\frac{d C_{D S}}{d x} V_{D S} \dot{x}(t)=C_{o} \frac{d V_{\text {out }}}{d t}$

Combining equations 0,0 and 0 , the expression of $E_{e}(t)$ becomes:

$$
\begin{gathered}
(\mathrm{Eq} 20) E_{e}(t)=\frac{C_{g}}{2} V_{\text {in }}{ }^{2}+\frac{C_{D S}}{2} V_{D S}{ }^{2}+\frac{C_{o}}{2} V_{\text {out }}{ }^{2}+\int\left(\frac{d C_{g}}{d x} V_{\text {in }}{ }^{2}+\frac{d C_{D S}}{d x} V_{D S}{ }^{2}\right) \dot{x}(t) d t \\
=\frac{C_{g}}{2} V_{\text {in }}{ }^{2}+\frac{C_{o}}{2} V_{\text {out }}{ }^{2}+\frac{C_{D S}}{2} V_{D S}{ }^{2}+E_{m}=E_{C g}+E_{C o}+E_{C D S}+E_{m}
\end{gathered}
$$

The first three terms of 0 represent the electrostatic energy stored in the capacitances $C_{g}, C_{o}$ and $C_{D S}$ respectively. The last term $E_{m}$ represents the mechanical energy. In other words, the electrical energy supplying a capacitive transducer is converted in two parts, the electrostatic energy stored in the variable capacitance and the output mechanical energy [27][28].

We have investigated the energy conversion and losses in the CAL buffer shown in Figure $4 \mathrm{a}$, and loaded by a logical sequence $V_{i n}$ (101) (Figure 6b). The variations of $\hat{C}_{D S}$ and $\hat{C}_{g}$, the non-dimensional values of $C_{D S}$ and $C_{g}$ (see Table 1) are shown in Figure 6c. During the Wait interval, $V_{\text {in }}$ increases and a large part of the electrical input energy $E_{\text {in }}$ is stored in $C_{g}$ (we call it $E_{C g}$ ). The rest is converted into the mechanical energy $E_{m}$ : part of it is stored in the mechanical springs that moves the body, leading to an increase of $C_{g}$ and $C_{D S}$ until they reach their maximum values. The remaining energy is converted into kinetic energy and part of it is lost during the impact between the drain and the source electrodes (see the oscillations of $\hat{C}_{D S}$ and $\hat{C}_{g}$ in Figure $6 \mathrm{c}$ ). During the Evaluate interval, $V_{P C}$ increases, generating an electrical input energy $E_{P C}$. However $C_{g}$ and $C_{D S}$ remain maximum and there is no electromechanical energy conversion: all the electrical energy $E_{P C}$ is stored in $C_{D S}$ and $C_{o}$.

During the Hold interval, $V_{i n}$ decreases but the capacitances $C_{D S}$ and $C_{g}$ do not change, as explained previously: only $E_{C g}$ is recovered by $V_{i n}$. During the Recovery interval, both electrical energies stored in $C_{D S}$ and $C_{o}$ are recovered by $V_{P C}$, and $C_{D S}$ remains constant for a while.

The electrical energy necessary to supply the buffer is not fully recovered: some energy is dissipated by damping losses and during the impact. Figure $6 \mathrm{~d}$ shows the evaluation of the 
non-dimensional electric energy $\hat{E}_{\text {in }}$ produced by the source $V_{i n}$, the energy $\hat{E}_{C g}$ stored in $C_{g}$, the energy $\hat{E}_{P C}$ produced by the source $V_{P C}$ and the energy $\left(\widehat{E}_{C_{D S}}+\widehat{E}_{C_{o}}\right)$ stored in both capacitances $C_{D S}$ and $C_{o}$. During one cycle, with the dimension in table 2 corresponding to a realistic device of $25 \mu \mathrm{m}^{2}$, the dissipated energy is equal to $0.9 \mathrm{fJ}$. However, most of the input energy $\left(\widehat{E}_{i n}+\widehat{E}_{P}\right)$ is recovered: the ratio between the recovered and the input energy is $89 \%$.

a.

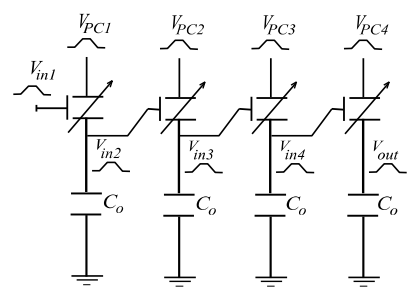

b.

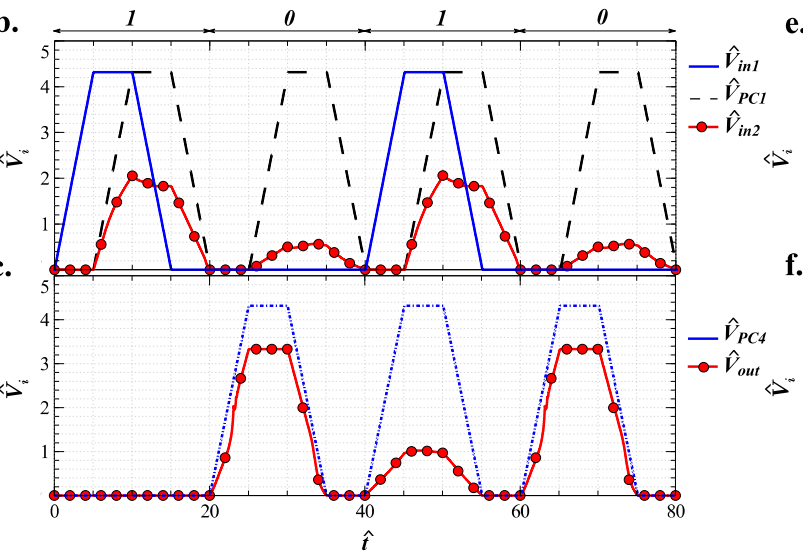

d.

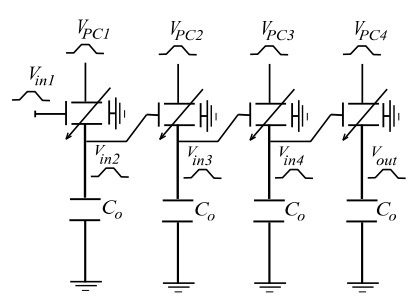

e.

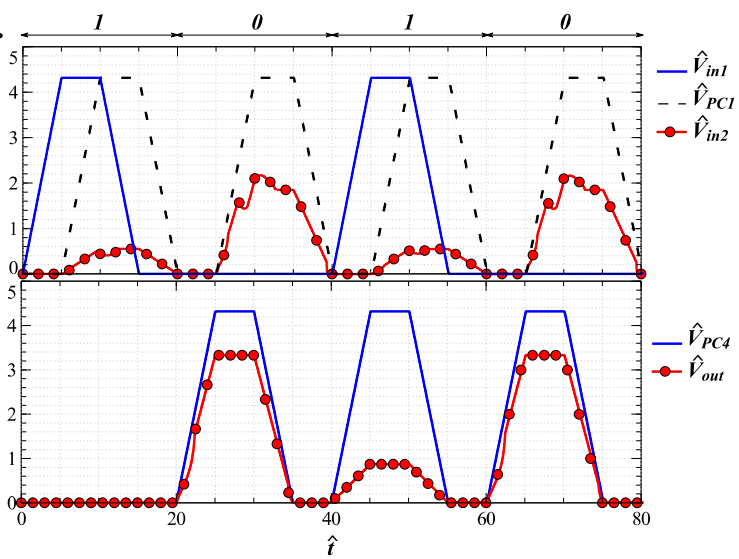

Figure 7. Experiments of cascability. a,d) Tested circuits made of 4 cascaded buffers/inverters. b,c) Evolution of voltages in cascaded buffers. e,f) Evolution of voltages in cascaded inverters.

\section{c) Cascability}

Now we consider the circuit composed of four cascaded CAL buffers and inverters. The buffer and inverter chain circuits are presented in Figure 7a,d respectively. Each gate is supplied by a PC, and the subsequent PCs are delayed by a quarter of period in order to satisfy the adiabatic conditions. The dynamic behavior of the cascaded buffers and inverters is given by the following non-dimensional equation:

$$
\begin{gathered}
\left(\text { Eq 21) } \ddot{\hat{x}}_{\mathrm{i}}+\lambda \dot{\hat{x}}_{\mathrm{i}}+\hat{x}_{\mathrm{i}}-\hat{V}_{i n, i}{ }^{2}\left[1+\frac{1}{\left(\beta_{1}-\hat{x}_{\mathrm{i}}^{2}\right)^{2}}+\frac{\gamma}{\left(\beta_{2}+\hat{x}^{0.75}\right.}\right]+\alpha\left(\alpha_{1} \widehat{V}_{D S, i}{ }^{2}\left[\frac{1+\beta_{4}\left(\beta_{5}+\hat{x}_{\mathrm{i}}\right)}{\left(\beta_{5}+\hat{x}_{\mathrm{i}}\right)^{2}}\right]-\right.\right. \\
\left.\alpha_{2} \widehat{V}_{P C, i}{ }^{2}\left[\frac{1+\beta_{4}\left(\beta_{3}-\hat{x}_{\mathrm{i}}\right)}{\left(\beta_{3}-\hat{x}_{\mathrm{i}}\right)^{2}}\right]\right)
\end{gathered}
$$

where $i$ refers to the position of the gate in the chain, $\left(\alpha_{1}=-1\right.$ and $\alpha_{2}=0$, for PVC configuration and $\alpha_{1}=\alpha_{2}=1$ for NVC) and:

$$
\text { (Eq 22) } \widehat{V}_{i n, i \geq 2}=\frac{\widehat{V}_{P C, i-1} C_{D S, i-1}}{C_{D S, i-1}+C_{g, i}+C_{o}},
$$




$$
\text { (Eq 23) } \widehat{V}_{D S, i}=\widehat{V}_{P C, i}\left(C_{g, i+1}+C_{o}\right) /\left(C_{D S, i}+C_{g, i+1}+C_{o}\right) .
$$

We studied the dynamical response of the cascaded elements to an input logic sequence $V_{i n 1}$ (1010). Figure $7 \mathrm{~b}$, e depict the dynamic output of the first gate for the buffer and inverter series. The logic state at the $4^{\text {th }}$ gate for the buffer and inverter series are shown in Figure $7 \mathrm{c}, \mathrm{f}$ respectively. The high (1) level corresponds to $77 \%$ of the PC's maximum voltage (equal to $1.38 \mathrm{~V}$ ) for both buffers and inverters chains, and the low (0) level corresponds to $23 \%$ and $20 \%$ of the PC for the buffers and the inverters chains respectively.

\section{Conclusion}

We have presented novel four-terminal variable capacitors (FTVC) based on silicon nano/micro technologies that can be used in Capacitive Adiabatic Logic. The FTVC intends to replace the field effect transistor (FET) in adiabatic logic in order to drastically reduce the energy consumption by avoiding the static losses and the non-adiabatic dissipation in classical CMOS circuits. It is a better alternative than nanorelays, also envisaged in adiabatic logic using electromechanical devices, because of the absence of resistive contact.

The electromechanical simulations demonstrated that with only two types of FTVC, it is possible to implement basic adiabatic logic functions as inverters and buffers. We also demonstrated for the first time the cascability of both class of devices. During one cycle, a buffer gate of $10 \times 2.5 \mu \mathrm{m}^{2}$, dissipated $0.9 \mathrm{fJ}$, which is in the same order of the energy dissipated by nano-scale FET transistors. However, it scales in the cube of the size. Gaining one order of magnitude in volume will allow to beat the dissipation of the state of the art of CMOS circuits: the simulations shows that a buffer gate of $1 \times 0.25 \mu \mathrm{m}^{2}$ would dissipate $0.7 \mathrm{aJ}$.

The simulations presented in this paper are performed with dimensions of devices that can be fabricated with classical MEMS and IC technologies. The characteristic dimension is 40 $\mathrm{nm}$ for both structures and gaps, and is still possible to achieve using photolithography as in last generations of IC technologies. In addition, there is much less steps in the fabrication process that for ICs and it does not require any expensive material. Reducing even more the dimensions will need the use of e-beam lithography, which is currently still quite expensive because of the longer time required for this step. Etching gaps in silicon at the nanometer scale would be also a challenge. However, reducing the size of the etching areas is very favorable to Deep Reactive Ion Etching of silicon because of an increase of the etching aspect-ratio (AR) with small dimensions: for instance in [29], Parasuraman et al have performed an AR of 125 for a gap of $35 \mathrm{~nm}$.

When adiabatic conditions are satisfied, most of the provided energy (89\%) is recovered after one cycle. This is a bit less than the non-contact device we have presented in [19]. However, the logic state differentiation has been dramatically improved from a few percents to more than $50 \%$. This is a considerable advantage for a practical implementation. 


\section{Aknowledgement}

The authors acknowledge financial support from the French Research National Agency (ANR) through the contract \#ANR-15-CE24-0013-02.

\section{References}

[1] S. Houri, G. Billiot, M. Belleville, A. Valentian, H. Fanet, Limits of CMOS Technology and Interest of NEMS Relays for Adiabatic Logic Applications, IEEE TCAS , 62 (2015) 1546-1554.

[2] A.P. Chandrakasan, S. Sheng, R.W. Brodersen, Low power CMOS digital design, IEEE Journal of Solid-State Circuits, 27 (1992) 473-484.

[3] A. Bellaouar, M. Elmarsry, Low Power digital VLSI Design, Circuits and Systems, 1995.

[4] K. Roy, S. Prasad, Low-power CMOS VLSI Circuit Design, Wiley, 2000.

[5] E. Pop, Energy Dissipation and Transport in Nanoscale Devices, Nano Research, 3 (2010) $147-$ 169.

[6] P. Teichmann, Adiabatic logic: future trend and system level perspective, Springer Science in Advanced Microelectronics, 2012.

[7] R. Landauer, "Irreversibility and Heat Generation in the Computing Process", IBM J. Res. Dev, 5 (1961) 183-191

[8] M. Madami, D. Chiuchiù, G. Carlotti, L. Gammaitoni, Fundamental energy limits in the physics of nanomagnetic binary switches, Nano Energy, 15 (2015) 313-320.

[9] H. Fanet, Ultra Low Power Electronics and Adiabatic Solutions, Electronics Engineering Series, ISTE / Wiley edition, 2016 - DOI: 10.1002/9781119006541

[10] Y. Moon, D.K Jeong, An Efficient Charge Recovery logic Circuit, IEEE J. Solid State Circuits, 31(1996) 514-522.

[11] S. Paul , A.M. Schlaffer, J.A. Nossek, Optimal charging of capacitors, IEEE Transactions on Circuits and Systems I: Fundamental Theory and Applications, 47 (2000) 1009-1016.

[12] O.Y. Loh, H.D. Espinosa, Nanoelectromechanical contact switches, Nature Nanotechnology, 7 (2012) 283-295.

[13] Z. Yi, J. Guo, Y. Chen, H. Zhang, S. Zhang, G. Xu, M. Yu and P. Cui, "Vertical, capacitive microelectromechanical switches produced via direct writing of copper wires", Microsystems \& Nanoengineering (2016) 216010 - doi: 10.1038/micronano.2016.10

[14] H. Kam, T-J. King Liu, V. Stojanovic, D. Markovic, E. Alon, Design optimization and scaling of MEM relays for ultra-low power digital logic, IEEE Trans. Electron Devices , 58 ( 2011) 236250.

[15] C. Pawashe, K. Lin, K.J. Kuhn, Scaling limits of electrostatic nanorelays, IEEE Transactions on Electron Devices, 60 (2013) 2936-2942.

[16] M. Spencer, F. Chen, C. C. Wang, R. Nathanael, H. Fariborzi, A. Gupta, H. Kam, V. Pott, J. Jeon, T-J. King Liu, D. Markovic, E. Alon, V. Stojanovic, Demonstration of Integrated Micro-ElectroMechanical Relay Circuits for VLSI Applications, IEEE Journal of Solid-State Circuits, 4 (2011) 308-320.

[17] S. Houri, C. Poulain, A. Valentian, H. Fanet, Performance Limits of Nanoelectromechanical Switches (NEMS)-Based Adiabatic Logic Circuits, J. Low Power Electron, 3 (2013) 368-384.

[18] G. Pillonnet, H. Fanet, S. Houri, Adiabatic capacitive logic: a paradigm for low power logic, 
Circuits and Systems (ISCAS), 2017 IEEE International Symposium on, Baltimore, MD, USA, 28-31 May 2017.

[19] A. Galisultanov, Y. Perrin, H. Samaali, H. Fanet, P. Basset and G. Pillonnet, "Contactless fourterminal MEMS Variable Capacitor for Capacitive Adiabatic Logic" , IOP Smart Materials and Structures, vol. 25, no 8, 2018 - DOI:10.1088/1361-665X/aacac4

[20] C.H. Bennett, Logical reversibility of computation, IBM Journal of Research and Development, 17 (1973) 525-532.

[21] J.G. Koller, W.C. Athas, Adiabatic switching, low energey computting, and the physics of storing and erasing information, Physics and Computation, PhysComp '92., Workshop on, Dallas, TX, USA, USA, 2-4 Oct. 1992.

[22] N.P. Vander Meijs, J.T. Fokkema, VLSI circuit reconstruction from mask topology, Integration the VLSI Journal, 2 (1984) 85-119.

[23] S. D. Senturia, "Microsystem design", Springer US edition, 2001, DOI 10.1007/b117574

[24] H.B. Palmer, Capacitance of a parallel-plate capacitor by the Schwartz-Christoffel transformation, Transactions of the American Institute of Electrical Engineers, 56 ( 1937 ) $363-$ 366.

[25] A. Tazzoli, M. Barbato, Study of the actuation speed, bounces occurrences, and contact reliability of ohmic RF-MEMS switches . Microelectronics Reliability 50 , (2010)1604-1608.

[26] A. Peschot , C. Poulain , N. Bonifaci , O. Lesaint, . Contact Bounce Phenomena in a MEMS Switch . IEEE 58th Holm Conference on Electrical Contacts (Holm), (2012).

[27] T. B. Jones, N. G. Nenadic, Electromechanics and MEMS. Cambridge University Pres, 2013.

[28] G. M. Rebeiz, RF MEMS: theory, design, and technology, John Wiley \& Sons, 2004.

[29] J. Parasuraman, A. Summanwar, F. Marty, P. Basset, D. E. Angelescu, C. Bunel and T. Bourouina, "Very High Aspect Ratio Deep Reactive Ion Etching of Sub-micrometer Trenches in Silicon", Microelectronics Engineering, vol. 113, pp. 35-39, Jan. 2014 


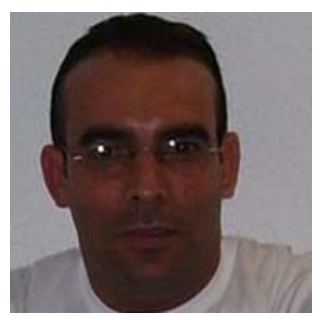

Dr. Hatem Samaali obtained his Ph.D. degree in Microelectronics from National Engineering School of Sfax (ENIS), Tunisia in 2011. He was a post-doc at ESIEE Paris, France, from 2016 to 2018 where he worked on NEMS-based adiabatic circuits. His current research topics include nonlinear dynamics in NEMS/MEMS Switch devices and Energy Harvesting.

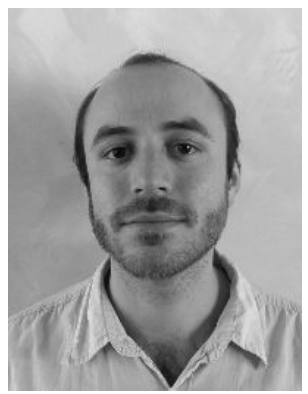

Dr. Yann Perrin was born in Fontainebleau, France, in 1989. He received his Master's degree in Nanophysics from the University of Paris-Sud, and his Ph.D in nanomagnetism from the University of Grenoble, in 2013 and 2016, respectively. He joined the CEA-LETI, France, in 2016 as a post-doctoral fellow. His research interests include energy-efficient circuits, and circuit design with novel devices such as micro electromechanical systems (MEMS).

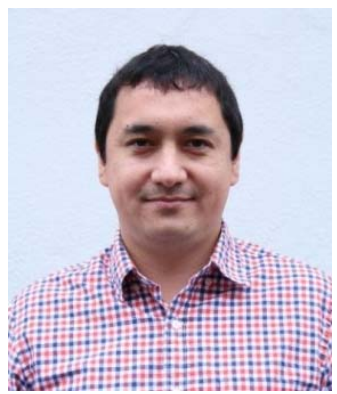

Dr. Ayrat Galisultanov received the Ph.D. degrees in Engineering from Avangard JSC, St. Petersburg, Russia in 2013 with research focus on SAW force sensor. From September 2009 to March 2015, he was an R\&D engineer at Avangard JSC. Ayrat Galisultanov was a postdoctoral researcher with FEMTO-ST Institute, Besançon, France prior to his current position of a postdoctoral researcher in CEA-LETI, Grenoble, France. His research interests include novel capacitive-based adiabatic logic devices, microelectromechanical systems, and SAW sensors.

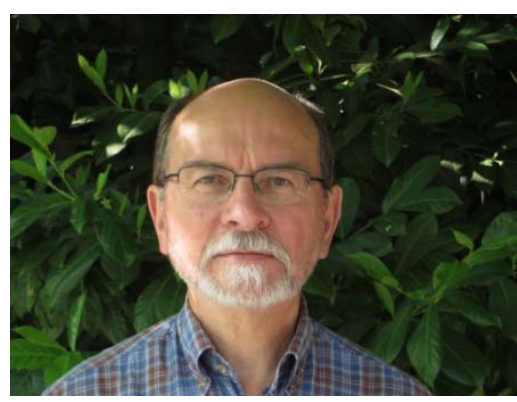

Hervé Fanet received in 1971 the engineer's diploma from the "Ecole Supérieure d'Electrricité". He was involved in detectors and electronic developments for high energy particle Physics. He joined 
CEA-LETI in 1996 and was in charge of medical imaging research and manager of integrated circuits design department. His current research topics include smart sensors and ultra-low power electronics. He has published 40 papers in journals and is author or co-author of five books

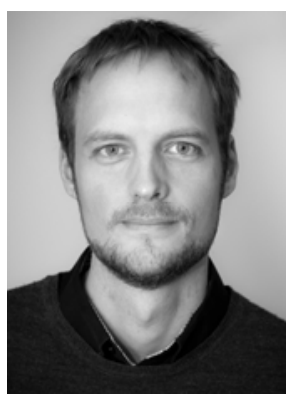

Dr.Gaël Pillonnet was born in Lyon, France, in 1981. He received his Master's degree in Electrical Engineering from CPE Lyon, France, in 2004, a PhD and habilitation degrees from INSA Lyon, France in 2007 and 2016, respectively. Following an early experience as analog designer in STMicroelectronics in 2008, he joined the University of Lyon in the Electrical Engineering department. During the 2011-12 academic year, he held a visiting researcher position at the University of California at Berkeley. Since 2013, he has been a full-time researcher at the CEA-LETI, a major French research institution. His research focuses on energy transfers in electronic devices such as power converter and amplifier, energy-recovery logic, mechatronics systems, scavenging interfaces.

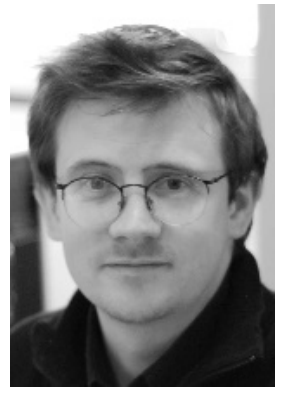

Dr. Philippe Basset is professor at Université Paris-Est / ESIEE Paris. He received his Ph.D from IEMN / University of Lille in 2003 in the areas of microelectronic and micro-electro-mechanicalsystems (MEMS). In 2004 he was a post-doc at CMU, Pittsburgh, USA and he joined ESIEE Paris in 2005. His current research interests include micro-power sources for autonomous MEMS and micro/nano-structuration of silicon. He serves in the International Steering Committee of the PowerMEMS conference since 2015 and is currently deputy director of the ESYCOM laboratory. 